\title{
Non-resonant Enhancement of Second-Harmonic Generation in a Dielectric Particle with a Nanostructured Nonlinear Metamaterial Shell
}

\author{
Joong Hwan Bahng1, Douglas Montjoy ${ }^{2}$, Saman Jahani ${ }^{1}$, Nicholas Kotov ${ }^{2}$, Alireza Marandi ${ }^{1}$ \\ ${ }^{I}$ Department of Electrical Engineering, California Institute of Technology, Pasadena, CA 91125 \\ ${ }^{2}$ Department of Chemical Engineering, University of Michigan, Ann Arbor, MI 48109 \\ joonghb@caltech.edu
}

\begin{abstract}
We demonstrate a new principle for realizing a miniaturized and scalable platform for nonlinear optics using dielectric particles with nanostructured nonlinear metamaterial shells. We show numerical and experimental results of enhanced second-harmonic generation in them. $\odot 2019$ The Author(s) OCIS codes: $160.4236,160.3918,190.3970$.
\end{abstract}

Engineered nanomaterials have gained attention for nonlinear optics (NLO) due to their strong field localization in the sub-wavelength scales and phase-matching free conditions. Their benefits have been recently demonstrated with remarkable NLO responses in the form of flat metamaterials and metasurfaces [1]. However, such precise nano-scale architectures do not liberate from complexities associated with the conventional nanofabrication techniques. On the other hand, nanostructures in colloidal/particulate platforms offer facile and scalable path to the development of freestanding sub-wavelength features and have recently attracted the NLO communities. However, so far, these studies have been limited to simple particles [2,3,4], and engineering metamaterials with them is largely unexplored.
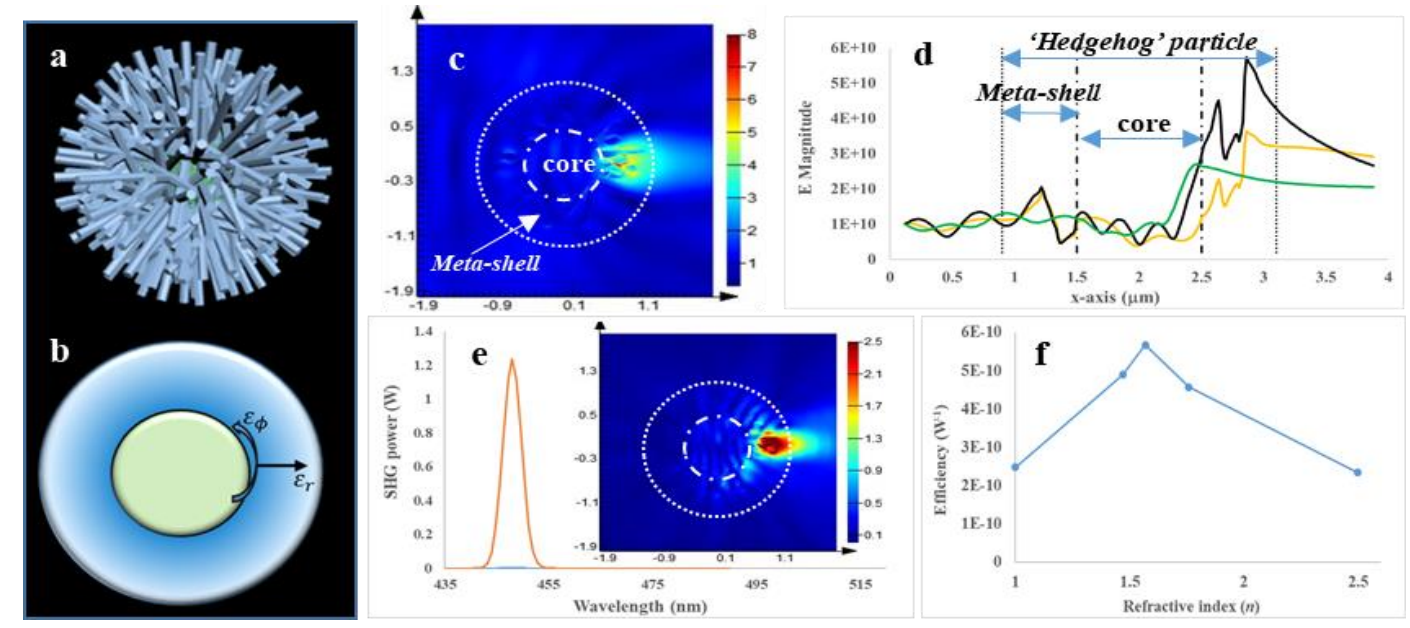

Figure 1: a) Schematics of the 'meta-shell' particles, also known as the 'hedgehog' particles; b) orthogonal orientation of high aspect ratio geometry in a spherical array renders a metamaterial shell with radial graded refractive index profile; c, d) concerted interplay between the electromagnetic responses of the core $\mu$-particle and that from the metamaterial shell architects strong photonic nano-jet within the shadow side within the metamaterial shell; e) FDTD simulations showing SHG at $\lambda=448 \mathrm{~nm}$ when the 'meta-shell' particles are pulsed with an arbitrary chosen $\lambda=896 \mathrm{~nm}$ at $100 \mathrm{fs}$ pulse-width, the inset shows the spatial and intensity profile of the SHG; $\mathbf{f}$ ) dependence of conversion efficiencies $\eta$ on the refractive index (n) of the core material: $n=1$ correspond to the 'meta-shell' particle without the core, $n=1.47$ correspond to silica, $n=1.57$ correspond to polystyrene, $\mathrm{n}=1.76$ correspond to alumina, $\mathrm{n}=2.5$ correspond to titania.

In this study, we took the nonlinear optics to the extreme sub-wavelength features arrayed in a 3D spherical construct morphed into micro/nano particles. As shown in the schematics, Figure 1a, the dielectric core microparticles are sculpted with shells of quadratically nonlinear metamaterials, which we call the 'Meta-Shell' particles. These particles are also known as 'Hedgehog' particles [5,6], Figure 2a, which are synthesized by orthogonally architecting high aspect-ratio $\mathrm{ZnO}$ nanospikes on to a low refractive index core dielectric micro-particle via a 
combination of hydrothermal and sonochemial processes. Since the spikes possess a relatively high $\chi^{2}$ coefficient [7], these 'meta-shell' particles will enable us to exploit the rich linear and nonlinear responses architected within and by the colloidal/particulate platform in synergy with that from the shell of metamaterials.
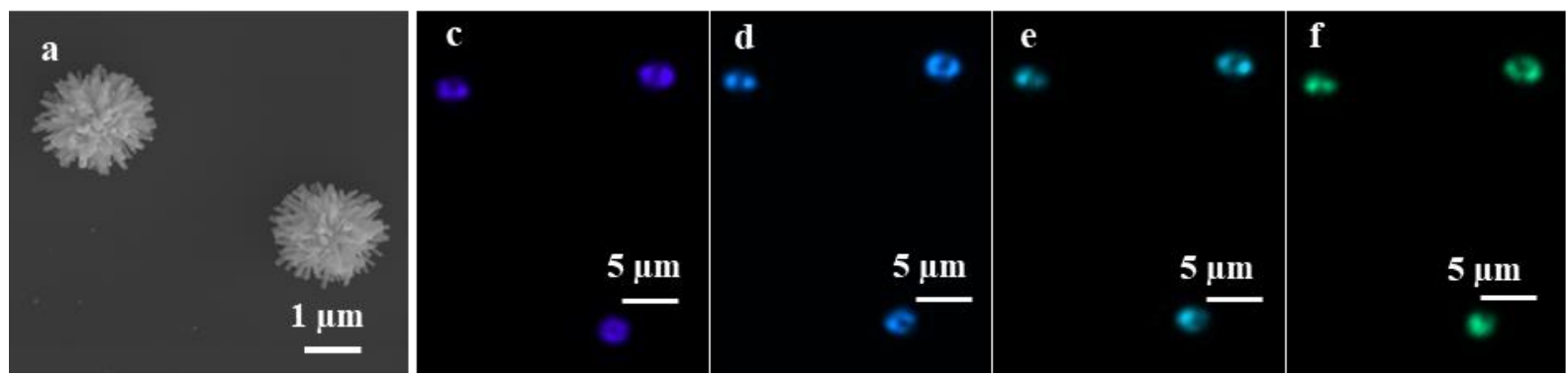

Figure 2: a) Scanning electron microscope image of the experimental construct of the 'meta-shell' particles. ZnO nanospikes having width of $120 \mathrm{~nm}$ and length of $600 \mathrm{~nm}$ are vertically sculpted on a polystyrene $\mu$-sphere; c-f) SHG images from the 'meta-shell' particles observed through confocal microscopy; SHG collected at (c) $\lambda=450 \mathrm{~nm}$, (d) $\lambda=470 \mathrm{~nm}$, (e) $\lambda=490$ $\mathrm{nm}$, (f) $\lambda=520 \mathrm{~nm}$ upon illumination at $\lambda=900 \mathrm{~nm}, 940 \mathrm{~nm}, 980 \mathrm{~nm}$ and $1020 \mathrm{~nm}$, respectively.

The FDTD simulations revealed approximately $10^{4}$-fold enhancement in the second harmonic generation (SHG) efficiency compared to a single $\mathrm{ZnO}$ spike in parallel orientation. This is due to concerted interplay between the two topographical features, the core and the metamaterial shell. The low refractive index core particle charters non-resonant refraction of incoming pump into a tightly confined and high intensity photonic nanojet within the shell of $\chi^{2}$ metamaterials, Figure 1c and 1d. Furthermore, the sub-wavelength arrangement of the metamaterial shell exhibits radially graded effective index due to the orthogonal orientation of high aspect-ratio geometry as schematized in Figure 1b, which further increases the incident light refraction into nanojet volume. Spatial coincidence can be seen between the high intensity nonlinear conversion and the photonic nanojet volume from the linear refraction, shown in the Figure 1e inset. Selection and design of the core material refractive indices plays an important role in optimizing the conversion efficiencies. Displacement of the intense nanoject away from the nonlinear metamaterial shell leads to leakage of field intensity into the core or the particle exterior, leading to lower conversion efficiencies, Figure 1f.

We have experimentally observed strong enhancements of SHG in a variety of 'meta-shell' particles. Figure 2a shows scanning electron microscope image of typical 'meta-shell' particles, and Figure 2c-f show the SHG images of the 'meta-shell' particles captured with a confocal microscope. Bearing in mind that the current generation of 'meta-shell' particles enable a non-resonant form SHG enhancement, superior performances may be achieved by assigning geometrical dimensions that coincide with the Mie resonant conditions for both the fundamental pump and the SHG. The 'meta-shell' particles boast procedurally simple, reproducible and versatile synthetic protocol with ease in which a consortium of materials geometries and constitutive properties may be designated.

\section{References}

[1] S. Jahani, Z. Jacob, “All-dielectric metamaterials”, Nat. Nanotechnology 11, 23 (2016).

[2] E. Kim, A. Steinbrück, M. Buscaglia, V. Buscaglia, T. Pertsch, R. Grange, "Second-Harmonic Generation of Single BaTiO3 Nanoparticles down to $22 \mathrm{~nm}$ diameter", ACS Nano 7 (6), 5343, (2013)

[3] F. Dutto, C. Raillon, K. Schenk, A. Radenovich, "Nonlinear Optical Response in Single Alkaline Niobate Nanowires", Nanoletters, 11 (6), 2517, (2011)

[4] P. Ray, "Size and Shape Dependent Second Order Nonlinear Optical Properties of Nanomaterials and Their Application in Biological and Chemical Sensing", Chem. Rev. 110, 5332, (2010)

[5] J. Bahng, B. Yeom, Y. Wang, S. Tung, J. Hoff, N. kotov, "Anomalous dispersions of 'hedgehog' particles", Nature 517, 596 (2015)

[6] D. Montjoy, J. Bahng, A. Eskafi, H. Hou, N. kotov, "Omnidispersible Hedgehog Particles with Multilayer Coatings for Multiplexed Biosensing", J. Am. Chem. Soc. 140 (25), 7835 (2018)

[7] J. Johnson, H. Yan, R. Schaller, P. Petersen, P. Yang, R. Saykally, "Near-Field Imaging of Nonlinear Optical Mixing in Single Zinc Oxide Nanowire", Nanoletters, 2 (4), 279, (2002) 